If $p=q+1$, replace (21) by

$$
\begin{aligned}
& {\left[\left(2 \alpha_{k}-\beta_{q}\right)(1-x)+(A-B) x\right] F} \\
& =\alpha_{k}(1-x) F\left(\alpha_{k}+\right)+\left(\alpha_{k}-\beta_{q}\right) F\left(\alpha_{k}-\right) \\
& \quad-x \sum_{j=1}^{q-1} V_{j, k} F\left(\beta_{j}+\right) ; \quad k=1,2, \cdots, p .
\end{aligned}
$$

UNIVERSITY OF MichigAN

\title{
ON THE GROWTH OF THE SOLUTIONS OF ORDINARY DIFFERENTIAL EQUATIONS
}

\section{A. ROSENBLATT}

In a recent paper, ${ }^{1} \mathrm{~N}$. Levinson gave four theorems concerning the behaviour of the solutions of the differential equation of elastic vibrations

$$
d^{2} x / d t^{2}+\phi(t) x=0
$$

as $t \rightarrow+\infty$. It is the purpose of this note to give generalizations of the Theorems I and III of Levinson by making use of certain inequalities concerning homogeneous equations of the first order

$$
\frac{d x_{i}}{d t}+\sum_{k=1}^{n} a_{i k} x_{k}=0, \quad i=1, \cdots, n .
$$

Theorems I and III of Levinson run as follows:

TheOREM I. If $\alpha(t)$ denotes the integral

$$
\alpha(t)=\int_{0}^{t}\left|\phi(t)-c^{2}\right| d t
$$

then

$$
x(t)=O\{\exp (\alpha(t) / 2 c)\} .
$$

THEOREM III. If $\alpha(t)$ is $O(t)$ then

$$
\limsup _{t \rightarrow \infty}|x(t) \exp (\alpha(t) / 2 c)|>0 \text {. }
$$

Received by the editors January 4, 1945.

1 The growth of the solutions of a differential equation, Duke Math. J. vol. 8 (1941) pp. 1-11. 
The Polish mathematician Z. Butlewski in a paper written in Polish $^{2}$ sets

$$
\begin{gathered}
r=\left(\sum_{1}^{n} x_{i}^{2}\right)^{1 / 2}, \\
\phi(t)=\sum_{1}^{n} a_{i i}\left(\frac{x_{i}}{r}\right)^{2}+\sum_{i, k=1}^{n}\left(a_{i k}+a_{k i}\right) \frac{x_{i} x_{k}}{r^{2}},
\end{gathered}
$$

where $i \neq k, i<k$, and obtains immediately from (2)

$$
r=C \exp \left(-\int_{t_{0}}^{t} \phi(\tau) d \tau\right) .
$$

Setting

$$
\alpha_{i i}=\int_{t_{0}}^{t}\left|a_{i i}\right| d \tau, \quad \beta_{i j}=\int_{t_{0}}^{t}\left|a_{i j}+a_{j i}\right| d \tau,
$$

we have

$$
r \leqq C \exp \left(\sum_{1}^{n} \alpha_{i i}+\frac{1}{2} \sum_{i, j=1 ; i<j}^{n} \beta_{i j}\right)
$$

and from this we have the following theorem.

Theorem XVIII of ButLewskr. If $\alpha_{i i}<+\infty$ and $\beta_{i j}<+\infty$, all the systems $x_{i}, i=1, \cdots, n$, of solutions of (2) are bounded.

With the designation

$$
M(t)=\max \int_{t_{0}}^{t}\left|a_{i j}\right| d \tau,
$$

we have

$$
r \leqq C \exp (n M(t)) .
$$

We can complete Butlewski's theorem by remarking that

$$
r \geqq C \exp \left(-\sum_{1}^{n} \alpha_{i i}-\frac{1}{2} \sum_{i, j=1 ; i<j}^{n} \beta_{i j}\right),
$$

and

$$
r \geqq C \exp (-n M(t)) .
$$

2 O całkach rzeczywistych równań róžniczkowych zwyczajnych, Wiadomości Matematyczne vol. 44 (1937) pp. 17-81. 
In the particular case $n=2$ Butlewski introduces polar coordinates

$$
x_{1}=\rho \cos \phi, \quad x_{2}=\rho \sin \phi
$$

and obtains

$$
\begin{aligned}
\rho=C \exp \left(-\int_{t_{0}}^{t}\left\{a_{11} \cos ^{2} \phi+\left(a_{12}+a_{21}\right)\right.\right. & \sin \phi \cos \phi \\
& \left.\left.\left.+a_{22} \sin ^{2} \phi\right)\right\} d \tau\right) .
\end{aligned}
$$

The maximum of $F(\phi)=-\left\{a_{11} \cos ^{2} \phi+\left(a_{12}+a_{21}\right) \sin \phi \cos \phi\right.$ $\left.+a_{22} \sin ^{2} \phi\right\}$ is

$$
2^{-1}\left\{-a_{11}-a_{22}+\left(\left(a_{11}-a_{22}\right)^{2}+\left(a_{12}+a_{21}\right)^{2}\right)^{1 / 2}\right\}
$$

so that

$$
\begin{aligned}
\rho \leqq C \exp \left(\frac { 1 } { 2 } \int _ { t _ { 0 } } ^ { t } \left\{-a_{11}-a_{22}+\left(\left(a_{11}-a_{22}\right)^{2}\right.\right.\right. & \\
& \left.\left.\left.+\left(a_{12}+a_{21}\right)^{2}\right)^{1 / 2}\right\} d \tau\right) .
\end{aligned}
$$

Thus we obtain the following theorem.

TheOREM XIX of Butlewski. $x_{1}, x_{2}$ are limited if

$$
\left.\frac{1}{2} \int_{t_{0}}^{t}\left\{-a_{11}-a_{22}+\left(\left(a_{11}-a_{22}\right)^{2}+\left(a_{12}+a_{21}\right)^{2}\right)^{1 / 2}\right\} d \tau\right)
$$

is limited.

In the same manner we obtain

$$
\begin{aligned}
\rho \geqq C \exp \left(\frac { 1 } { 2 } \int _ { t _ { 0 } } ^ { t } \left\{-a_{11}-a_{22}-\left(\left(a_{11}-a_{22}\right)^{2}\right.\right.\right. \\
\left.\left.\left.+\left(a_{12}+a_{21}\right)^{2}\right)^{1 / 2}\right\} d \tau\right),
\end{aligned}
$$

completing Butlewski's results.

The linear differential equation

$$
x^{\prime \prime}+\psi(t) x^{\prime}+\phi(t) x=0
$$

with continuous $\phi$ and $\psi$ can be transformed into a system (2) by setting

$$
x^{\prime}=\lambda x_{2}, \quad x=x_{1},
$$


$\lambda \neq 0$, continuous in $t$. We obtain the system

$$
\frac{d x_{1}}{d t}-\lambda x_{2}=0, \quad \frac{d x_{2}}{d t}+\frac{\phi}{\lambda} x_{1}+\left(\frac{\lambda^{\prime}}{\lambda}+\psi\right) x_{2}=0,
$$

$a_{11}=0, a_{12}=-\lambda, a_{21}=\phi / \lambda, a_{22}=\lambda^{\prime} / \lambda+\psi$.

The inequalities (17), (19) now take the form

$$
\begin{array}{r}
\rho \leqq C \exp \left(\frac { 1 } { 2 } \int _ { t _ { 0 } } ^ { t } \left\{-\frac{\lambda^{\prime}}{\lambda}-\psi+\left(\left(\frac{\lambda^{\prime}}{\lambda}+\psi\right)^{2}\right.\right.\right. \\
\left.\left.\left.+\left(\frac{\phi}{\lambda}-\lambda\right)^{2}\right)^{1 / 2}\right\} d \tau\right), \\
\rho \geqq C \exp \left(\frac { 1 } { 2 } \int _ { t _ { 0 } } ^ { t } \left\{-\frac{\lambda^{\prime}}{\lambda}-\psi-\left(\left(\frac{\lambda^{\prime}}{\lambda}+\psi\right)^{2}\right.\right.\right. \\
\left.\left.\left.+\left(\frac{\phi}{\lambda}-\lambda\right)^{2}\right)^{1 / 2}\right\} d \tau\right) .
\end{array}
$$

Taking $\lambda=c$, Butlewski obtains

$$
\rho \leqq C \exp \left(\frac{1}{2} \int_{t_{0}}^{t}\left\{-\psi+\left(\psi^{2}+\left(\frac{\phi}{c}-c\right)^{2}\right)^{1 / 2}\right\} d \tau\right)
$$

and we can add the inequality

$$
\rho \geqq C \exp \left(\frac{1}{2} \int_{t_{0}}^{t}\left\{-\psi-\left(\psi^{2}+\left(\frac{\phi}{c}-c\right)^{2}\right)^{1 / 2}\right\} d \tau\right) .
$$

We shall now generalize Levinson's Theorem III for the equation (20).

We can suppose $x>0, x^{\prime}<0$, otherwise we should have an infinite number of values $t=t_{i}, i=1,2, \cdots ; t_{i} \rightarrow \infty$, with

$$
\left|x\left(t_{i}\right)\right| \geqq C \exp \left(\frac{1}{2} \int_{t_{0}}^{t}\left\{-\psi-\left(\psi^{2}+\left(\frac{\phi}{c}-c\right)^{2}\right)^{1 / 2}\right\} d \tau\right) .
$$

Consider the intervals $n \leqq t \leqq n+1$. We have

$$
x(n+1)-x(n)=\int_{n}^{n+1} x^{\prime}(t) d t,
$$

and denoting by $x_{n}^{\prime}$ the maximum of $x^{\prime}(t)$ in $\langle n, n+1\rangle$,

$$
x(n) \geqq-x_{n}^{\prime}, \quad x_{n}^{\prime}=x^{\prime}\left(t_{n}\right), \quad n \leqq t_{n} \leqq n+1 .
$$

We have 


$$
\begin{aligned}
x^{\prime}(n)-x_{n}^{\prime} & =\int_{t_{n}}^{n} x^{\prime \prime}(t) d t=-\int_{t_{n}}^{n}\left(\phi x+\psi x^{\prime}\right) d t \\
\left|x^{\prime}(n)\right| & \leqq x(n)+x(n) \int_{n}^{n+1}|\phi| d t+|\psi|_{\max } x(n) \\
& =x(n)\left\{1+\int_{n}^{n+1}|\phi| d t+|\psi|_{\max }\right\} .
\end{aligned}
$$

$|\psi|_{\max }$ is the maximum of $|\psi|$ in $n \leqq t \leqq n+1$.

We obtain

$$
x(n) \geqq \frac{C \exp \left(\frac{1}{2} \int_{t_{\theta}}^{t}\left\{-\psi-\left(\psi^{2}+\left(\frac{\phi}{c}-c\right)^{2}\right)^{1 / 2}\right\} d \tau\right)}{1+\frac{1}{c^{2}}\left(1+|\psi|_{\max }+\int_{n}^{n+1}|\phi| d t\right)} .
$$

The result is the following theorem.

THEOREM. There exist infinite values of $t=t_{i}, t_{i} \rightarrow \infty$, for which we have (27) if the following conditions are satisfied:

1. $\alpha(t)$ is of order $O(t)$.

2. $|\psi|$ is bounded.

UNIVERSITY OF SAN MARCoS 